\title{
Brief History of Protein Degradation and the Ubiquitin System
}

\author{
Avram Hershko
}

\section{1}

Introductory Remarks

The reader of this book may be impressed (and possibly overwhelmed) by the enormous recent progress in this field. The ubiquitin system is now known to be involved in basic biological processes, such as the control of cell division, signal transduction, regulation of transcription, DNA repair, quality control in the endoplasmic reticulum, stress response, induction of immune response and inflammation, apoptosis, embryonic development, and circadian clocks, to mention but a few. It has been implicated in diseases such as many types of cancer, neurodegenerative diseases (such as certain types of Parkinson's, Alzheimer's and Huntington's diseases), retroviral infections, certain types of hypertension, mental retardation, and cachexia associated with cancer, renal failure, or sepsis. New functions of ubiquitin and of ubiquitin-like proteins are being reported almost every month, and the number of publications in this field is increasing at an exponential (and bewildering!) rate. It may be therefore instructive to consider briefly the humble beginnings of this field, how significant progress was achieved, and also how at times progress was impeded by wrong dogmas. Important lessons can be learned from both achievements and failures in science.

1.2

\section{Protein Degradation - Does It Exist?}

In the first three decades of the twentieth century, a generally accepted theory of protein metabolism was that proposed by Folin [1]. Based on studies on the chemical composition of urine in humans fed protein-rich or protein-free diets, Folin proposed that there are two separate pathways of protein catabolism, which he called "endogenous" and "exogenous" types of protein catabolism. According to this concept, "exogenous" protein catabolism originates from dietary proteins, accounts for the major part of urea excreted under normal conditions, and shows wide variations according to dietary protein intake. By contrast, "endogenous" pro-

Protein Degradation. Edited by J. Mayer, A. Ciechanover, M. Rechsteiner Copyright (C) 2005 WILEY-VCH Verlag GmbH \& Co. KGaA, Weinheim ISBN: 3-527-30837-7 
2 1 Brief History of Protein Degradation and the Ubiquitin System

tein catabolism was thought to originate from tissue proteins, to be mainly represented by excreted creatinine, not to be affected by the amount of dietary protein intake, and to account for a minor part of nitrogenous compounds excreted in the urine. Because of the minor proportion of "endogenous" protein catabolism, it was thought that cellular proteins are predominantly stable, and only a small fraction resulting from "wear and tear" of tissue proteins is subject to catabolism [1].

In spite of its obviously wrong assumptions (such as that creatinine is the end product of protein catabolism), Folin's theory was widely accepted and cited in textbooks of biochemistry until the late thirties. At that time, a breakthrough in the field was achieved by the pioneering studies of Schoenheimer and co-workers, who introduced the extensive use of isotopically labeled compounds in biological studies. In a typical experiment [2], ${ }^{15} \mathrm{~N}$-labeled L-leucine was administered to well-fed rats, and the distribution of the isotope in excreta and in body tissues was examined. According to the concept of Folin, most exogenously administered leucine should have appeared in urinary waste products. This was not the case: less than one-third of the isotope was excreted in the urine, and most of it was found to be incorporated into tissue proteins [2]. Since the weight of the animals did not change during the experiment, it could be assumed that the mass and composition of body proteins also did not change. It was concluded, therefore, that newly incorporated amino acids must have replaced those in tissue proteins in a process of protein turnover. From these studies a new concept has emerged according to which cellular proteins, and some other body constituents, are in a dynamic state of constant and extensive renewal [3].

Schoenheimer's concept of the dynamic state of body proteins did not remain unchallenged. In 1955, Monod and co-workers studied the origin of amino acids utilized for the synthesis of newly induced $\beta$-galactosidase in growing E. coli [4]. Bacteria were first labeled with ${ }^{35} \mathrm{SO}_{4}{ }^{2-}$ and then were transferred to unlabeled medium containing the inducer methyl- $\beta$-D-thiogalactoside. Newly synthesized $\beta$ galactosidase was isolated and was found not to contain significant amounts of radioactivity. This result suggested that in growing E. coli, the degradation of most cellular proteins is negligibly slow, otherwise newly synthesized $\beta$-galactosidase would have contained ${ }^{35} \mathrm{~S}$-labeled amino acids originating from the degradation of pre-existing proteins. Instead of restricting these conclusions to the case of growing E. coli, the authors went on to generalize and proposed that cellular proteins are also stable in mammalian tissues. They furthermore suggested that in Schoenheimer's experiments, incorporation of amino acids into tissue proteins might be due to the replacement of cells lost by cell lysis or the replacement of secreted proteins [4]. This was, in effect, a return to Folin's dogma of static cellular proteins.

So great was the authority of Monod at that time that the dynamic state concept of Schoenheimer fell into disfavor, as judged by contemporary review articles [5]. Gradually, however, experimental evidence accumulated which refuted Monod's hypothesis. Using mammalian cells in culture, Eagle and co-workers carefully examined the problem of cellular protein turnover $v$ s. cell turnover or protein secretion [6]. In a variety of growing or resting cells in culture, cellular proteins were replaced at a high rate of approx. $1 \% \mathrm{~h}^{-1}$. This was due to true protein turnover 
and not to the replacement of secreted proteins or lysed cells, as indicated by the lack of significant amounts of labeled proteins in the culture medium. Further work in several laboratories has shown that protein degradation in animal cells is extensive and is highly selective. Thus, for example, abnormal proteins produced by the incorporation of some amino acid analogues or by certain mutations are selectively recognized and are rapidly degraded in cells [7]. However, it is not correct to state (as is written in some current articles) that, until recently, protein degradation was thought to be mainly a "garbage disposal" system to get rid of abnormal proteins. In the late sixties, it was already evident that normal proteins are also degraded in a highly selective mode. The half-lifetimes of different proteins range from several minutes to many days, and rapidly degraded normal proteins usually have important regulatory functions. These properties of intracellular protein degradation and the importance of this process in the control of the levels of specific proteins were summarized by Schimke and Doyle in 1970 [8].

In retrospect, one can only speculate why the concept of intracellular protein degradation was resisted for such a long time. It is possible that one reason was the difficulty in accepting the idea that cells carry out such a wasteful process. A substantial amount of energy is invested in the formation of peptide bonds in the process of protein synthesis, and all this energy is dissipated when the protein is degraded. A possible explanation is that energy expenditure is used to achieve regulation. Our current knowledge of some of the functions of the ubiquitin system is consistent with this notion.

\section{3}

\section{Discovery of the Role of Ubiquitin in Protein Degradation}

Although the basically important cellular functions of selective protein degradation became evident in the late sixties, the molecular mechanisms involved in this process remained unknown. I became interested in the problem of how proteins are degraded in cells when I was a postdoctoral fellow in the laboratory of Gordon Tomkins in 1969-71. Gordon was mainly interested at that time in the mechanisms by which steroid hormones induce the synthesis of specific proteins. His model system for this purpose was the regulation of the enzyme tyrosine aminotransferase (TAT) in cultured hepatoma cells. Like other regulatory proteins, TAT has a rapid degradation rate. I found at that time, quite by accident, that the degradation of TAT is blocked by inhibitors of cellular ATP production, such as fluoride or dinitrophenol [9]. These results confirmed and extended earlier findings of Simpson [10] on the energy-dependence of the liberation of amino acids from proteins in liver slices. Since ATP depletion also prevented the inactivation of the enzymatic activity of TAT, it was concluded that energy is required at an early step in the process of protein degradation [9].

I was very much impressed by the energy-dependence of intracellular protein degradation because it suggested the involvement of a novel mechanism, different from that of known proteolytic enzymes. One attractive possibility that I consid- 
\begin{tabular}{l|ll}
4 & 1 Brief History of Protein Degradation and the Ubiquitin System
\end{tabular}

ered was that proteins may be modified by some energy-dependent reaction prior to their degradation, and that such modification renders them susceptible to the action of some proteolytic enzyme [11]. To examine the existence of such (or any other) mechanism, a cell-free system was required, which faithfully reproduced energy-dependent protein degradation in the test tube, and which could be subjected to biochemical analysis. A cell-free ATP-dependent proteolytic system from reticulocyte lysates was first established by Etlinger and Goldberg [12]. Subsequently, my laboratory subjected this system to biochemical fractionation, with the aim of isolating its components and characterizing their mode of action. In this work, I was greatly helped by Aaron Ciechanover, who was my graduate student at that time. I have also received a lot of support, help, and great advice from Irwin Rose, in whose laboratory at Fox Chase Cancer Center I worked in a sabbatical year in 1978-79 and for many summers afterwards.

In the initial experiments, we resolved reticulocyte lysates on DEAE-cellulose into two crude fractions: Fraction 1, which contained proteins not adsorbed to the resin, and Fraction 2, which contained all proteins adsorbed to the resin and eluted with high salt. The original aim of this fractionation was to get rid of hemoglobin, which was known to be in Fraction 1, while most non-hemoglobin proteins of reticulocytes were known to be in Fraction 2. We found that neither fraction was active by itself, but ATP-dependent protein degradation could be reconstituted by combination of the two fractions [13]. The active component in Fraction 1 was a small, heat-stable protein; we have exploited its stability to heat treatment for its purification to near homogeneity. We termed this protein at that time APF-1, for ATP-dependent Proteolysis Factor 1 [13]. The identity of APF-1 with ubiquitin was established later by Wilkinson et al. [14], subsequent to the discovery in my laboratory of its covalent ligation to protein substrates, as described below.

The next question was what is the role of this small protein in ATP-dependent protein degradation. It looked smaller than most enzymes, so at first I thought that it might be a regulatory subunit of some enzyme (such as a protein kinase or an ATP-dependent protease) present in Fraction 2. To test this notion, we looked for the association of APF-1/ubiquitin with some protein in Fraction 2. For this purpose, purified radiolabeled APF-1/ubiquitin was incubated with Fraction 2 in the presence or absence of ATP, and subjected to gel filtration chromatography. A marked ATP-dependent association of APF-1/ubiquitin with high molecular weight material was observed [15]. It was very surprising to find that binding was covalent, as indicated by the resistance of the high molecular weight derivative to a variety of denaturing agents [15]. Subsequent work showed that proteins to which ubiquitin is bound are substrates of the ATP-dependent proteolytic system [16]. Based on these findings, we proposed in 1980 that proteins are targeted for degradation by covalent ligation to APF-1/ubiquitin and hypothesized that a protease exists that specifically degrades proteins ligated to ubiquitin [16]. Shortly afterwards, the identity of APF-1 with ubiquitin was established by Wilkinson et al. [14]. Ubiquitin was originally isolated by Goldstein and co-workers in a search for hormones from the thymus, but was subsequently found to be present in all tissues and eukaryotic organisms, hence its name [17]. The functions of ubiquitin were not 
1.4 Identification of Enzymes of the Ubiquitin-mediated Proteolytic System $\mid \mathbf{5}$

known, though it was discovered by Goldknopf, Busch, and co-workers that ubiquitin is conjugated to histone $2 \mathrm{~A}$ in an isopeptide linkage [18].

\section{4}

Identification of Enzymes of the Ubiquitin-mediated Proteolytic System

In subsequent work in my laboratory, we tried to isolate and characterize enzymes of the ubiquitin-mediated proteolytic system from Fraction 2 of reticulocytes, using a similar biochemical fractionation-reconstitution approach. Over a period of about ten years (1980-1990), we have identified eight different components in Fraction 2, all of which were required for ubiquitin-ATP-dependent protein degradation. Three of these are involved in the conjugation of ubiquitin to protein substrates. These are the ubiquitin-activating enzyme E1 [19], ubiquitin-carrier protein E2 [20] and ubiquitin-protein ligase E3 [20]. We found that E1 carries out the ATP-dependent activation of the carboxy-terminal glycine residue of ubiquitin [21] by the formation of ubiquitin adenylate, followed by the transfer of activated ubiquitin to a thiol site of E1 with the formation of a thiolester linkage [19, 20]. Activated ubiquitin is transferred to a thiol site of E2 by transacylation, and is then further transferred to an amino group of the protein substrate in a reaction that requires E3 [20]. All three types of enzyme were purified by affinity chromatography on ubiquitinSepharose [20]. The terms E1, E2, and E3 were suggested by Ernie Rose; "E" stood for enzyme, and not eluate, as stated in some articles. We found that the role of E3 is to specifically bind specific protein substrates [22]. Building on this observation, it was proposed that the selectivity of ubiquitin-mediated protein degradation is mainly determined by the substrate specificity of different E3 enzymes [23]. This notion was verified by subsequent work in many laboratories on the selective action of a large number of different E3 enzymes on their specific protein substrates.

Three other components that my laboratory has identified and partially purified from Fraction 2 of reticulocytes, termed CF1-CF3, are involved in the degradation of proteins ligated to ubiquitin [24]. These are apparently subcomplexes of the 26S proteasome, a large ATP-dependent protease complex first described by Rechsteiner and co-workers [25]. CF3 is identical to the 20S proteasome core particle [26], while CF1 and CF2 may be similar to the "base" and "lid" subcomplexes of the $19 \mathrm{~S}$ regulatory particle of the $26 \mathrm{~S}$ proteasome, described more recently by the Finley laboratory [27]. In hindsight, the reason for finding subcomplexes, rather than the complete 26S complex in Fraction 2 was technical: we have routinely prepared Fraction 2 from ATP-depleted reticulocytes [20], under which conditions the $26 \mathrm{~S}$ proteasome dissociates to its subcomplexes. We found that incubation of the three subcomplexes in the presence of ATP promotes their assembly to the $26 \mathrm{~S}$ proteasome $[24,26]$. The role of ATP in the assembly of the 26S proteasome complex remains unknown.

The last two enzymatic activities that we have described in reticulocytes are ubiquitin C-terminal isopeptidases, which act at the final stages of the ubiquitin proteo- 
6 6 1 Brief History of Protein Degradation and the Ubiquitin System

lytic pathway to release free and reusable ubiquitin from intermediary degradation products. One is an enzyme called isopeptidase $\mathrm{T}$, which preferentially cleaves ubiquitin-Lys ${ }^{48}$-ubiquitin linkages in polyubiquitin chains [28]. Its main function appears to be the disassembly of polyubiquitin chain remnants following proteolysis of the protein substrate moiety of ubiquitin-protein conjugates by the 26S proteasome complex. Another is a ubiquitin-C-terminal hydrolase that is an integral part of $26 \mathrm{~S}$ proteasome complex [29]. Its role appears to be to release ubiquitin from linkage to the protein substrate at the final stages of the action of the 26S proteasome. Unlike most ubiquitin-C-terminal hydrolases, this isopeptidase is not inhibited by ubiquitin aldehyde, but is inhibited by the heavy metal chelator o-phenanthroline [29]. It appears to be similar to the Rpn11 metalloprotease subunit of the lid subcomplex, which has been recently identified by the Deshaies laboratory and shown to be essential for substrate deubiquitination and degradation [30].

\section{5}

\section{Discovery of Some Basic Cellular Functions of the Ubiquitin System}

The discovery of the basic biochemistry of ubiquitin-mediated protein degradation opened up the way for significant further progress in the elucidation of the roles of this system in a large variety of biological processes. Such further progress required the additional approaches of molecular genetics and cell biology. Thus, the first indication of the role of the ubiquitin system in cell cycle control was the discovery by Varshavsky and co-workers that the ts 85 mammalian cell line, which fails to enter mitosis at the restrictive temperature, is defective in the ubiquitinactivating enzyme E1 [31]. The cloning of various genes of the ubiquitin system in yeast by the same laboratory led to insights into the roles of the polyubiquitin gene in stress response [32] and to the identification of the product of the DNA repair gene $R A D 6$ as an E2 protein [33]. Shortly afterwards, another E2 protein was identified as the product of the CDC34 gene, known to be involved in the $\mathrm{G} 1 \rightarrow \mathrm{S}$ transition in the cell cycle [34]. These early studies on the molecular genetics of the ubiquitin system initiated an avalanche of rapid progress in this field by many laboratories.

The entry of molecular genetics into the ubiquitin field did not signal the end of the usefulness of biochemical approaches. A good example of the power of the combination of biochemistry with genetics is the discovery of the Anaphase Promoting Complex/Cyclosome (APC/C), a large multisubunit ubiquitin ligase essential for exit from mitosis by the degradation of mitotic regulators such as cyclin B. In 1983, Hunt and co-workers discovered cyclin B, the first cyclin, as a protein that is destroyed at the end of each cell cycle in early embryos of marine invertebrates [35]. This discovery not only opened up a new era in cell cycle research, but also kindled interest in the problems of what is the machinery that targets cyclin B for degradation, and why does it act only at the end of mitosis? Though researchers of the cell cycle were at that time searching for a putative "cyclin protease", I thought that a specific ubiquitin ligase might exist that acts on cyclin B only at the end of 
mitosis. In 1991, independent work from the laboratory of Kirschner [36] and from my laboratory [37] showed that cyclin B is degraded by the ubiquitin system. Both laboratories employed biochemical approaches, using cell-free systems from early embryos of frogs [36] and clams [37]. Initial fractionation of the system in my laboratory [38] showed that in addition to E1, two novel components were required to reconstitute cyclin-ubiquitin ligation. These were a specific E2, termed E2-C, and an E3-like activity, which, in clam extracts, was associated with particulate material. In 1995, rapid progress in this system took place due to the convergence of information from biochemical experiments with genetic analysis in yeast. In work done in collaboration with Joan Ruderman, we solubilized the E3-like activity and partially purified and characterized it [39]. It was found to be a large $(\sim 1500 \mathrm{kDa})$ complex, which has cyclin-ubiquitin ligase activity. The activity of this enzyme is regulated in the cell cycle: it is inactive in the interphase and becomes active at the end of mitosis by phosphorylation. We called this complex the cyclosome, to denote its large size and important roles in cell-cycle regulation [39]. A similar complex was isolated from frog extracts at the same time by the Kirschner lab, and was called the Anaphase Promoting Complex [40]. The identification of subunits of the APC/C was made possible by work from the Nasmyth laboratory, who used an elegant genetic screen to identify yeast genes required for the proteolysis of cyclin B [41]. The products of some of these genes, CDC16, CDC23 and CDC27, had been previously shown to be required for the onset of anaphase in budding and fission yeasts. Thus, the genetic work also proved the relevance of the biochemical results on APC/C to its role in exit from mitosis in cells. Subsequent work by several groups showed that APC/C is also involved in the degradation of some other important mitotic regulators, such as securin, an inhibitor of anaphase onset (reviewed in Ref. [42]). In addition, the APC/C is the target of the spindle assembly checkpoint system, a surveillance mechanism that allows sister chromatid separation only after all chromatids have been properly attached to the mitotic spindle [43].

1.6

Concluding Remarks

Several lessons can be learned from our story. One is not to accept authority in science. Monod's statement that there is no protein turnover in animal cells should not have been accepted without examination of the assumptions on which the statement was based. A second is that if you believe that you have a biologically important problem to study, you should pursue it, even if very few other researchers are interested in it. At the beginning, very few scientists were interested in the ubiquitin system (and some of the few who knew about it thought it was all wrong), but being obstinate was rewarding in the long run. If everyone only worked on subjects that are in the current mainstream of science, very few new fields would be discovered. The third lesson, which I keep reiterating with the hope of convincing a few young scientists, is the continued importance of bio- 
8 1 Brief History of Protein Degradation and the Ubiquitin System

chemistry in biomedical research. The ubiquitin system could not have been discovered without the use of biochemical approaches, and biochemistry continues to be essential, in combination with molecular genetics and cell biology, in unraveling the myriad cellular functions of this system and their underlying molecular mechanisms.

\section{References}

1 Folin, O. Am. J. Physiol. 1905, 13, 66116.

2 Schoenheimer, R., Ratner, S. and Rittenberg, D. J. Biol. Chem. 1939, 130, 703-732.

3 Schoenheimer, R. The Dynamic State of Body Constituents, Harvard University Press, Cambridge, MA, 1942.

4 Hogness, D. S., Cohn, M. and Monod. J. Biochim. Biophys. Acta 1955, 16, 99-116.

5 Kamin, H. and Handler, P. Annu. Rev. Biochem. 1957, 26, 419-490.

6 Eagle, H., Piez, K. A., Fleischman, R. and Oyama, V. I. J. Biol. Chem. 1959, 234, 52-597.

7 Rabinowitz, M. and Fisher, J. M. Biochim. Biophys. Acta 1964, 91, 313322.

8 Schimke, R. T. and Doyle, D. Annu. Rev. Biochem. 1970, 39, 929-976.

9 Hershko, A. and Tomkins, G. M. J. Biol. Chem. 1971, 246, 710-714.

10 Simpson, M. V. J. Biol. Chem. 1953, 201, 143-154.

11 Hershko, A. Regulation of Gene Expression, Harris, M. and Thompson, B., eds., U.S. Government Printing Office, Washington, DC, 1974, pp. 85-94.

12 Etlinger, J. D. and Goldberg, A. L. Proc. Natl. Acad, Sci. USA 1977, 74, 54-58.

13 Ciechanover, A., Hod, Y. and Hershro, A. Biochem. Biophys. Res. Commun. 1978, 81, 1100-1105.

14 Wilkinson, K. D., Urban, M. K. and HaAs, A. L. J. Biol. Chem. 1980, 255, 7529-7532.

15 Ciechanover, A., Heller, H., Elias, S., HaAs, A. L. and Hershro, A. Proc. Natl. Acad. Sci. USA 1980, 77, 13651368.
16 Hershio, A., Ciechanover, A., Heller, H., HaAs, A. L. and Rose, I. A. Proc. Natl. Acad. Sci. USA 1980, 77, 1783-1786.

17 Goldstein, G., Scheid, M., Hammerling, U., Boyse, E. A., SChlesinger, D. H. and Niall, H. D. Proc. Natl. Acad. Sci. USA 1975, 72, 11-15.

18 Goldknopf, I. L. and Busch, H. Proc. Natl. Acad. Sci. USA 1977, 74, 864868.

19 Ciechanover, A., Heller, H., KatzEtzion, R. and Hershko, A. Proc. Natl. Acad. Sci. USA 1981, 78, 761765.

20 Hershko, A., Heller, H., Elias, S. and Ciechanover, A. J. Biol. Chem. 1983, 258, 8206-8214.

21 Hershro, A., Ciechanover, A. and Rose, I. A. J. Biol. Chem. 1981, 256, 1525-1528.

22 Hershko, A., Heller, H., Eytan, E. and Reiss, Y. J. Biol. Chem. 1986, 261, 11992-11999.

23 Hershko, A. J. Biol. Chem. 1988, 263, 15237-15240.

24 Ganoth, D., Leshinsky, E., Eytan, E. and Hersh ko, A. J. Biol. Chem. 1988, 263, 12412-12419.

25 Hough, R., Pratt, G. and Rechsteiner, M. J. Biol. Chem. 1986, 261, 2400-2408.

26 Eytan, E., Ganoth, D., Armon, T. and Hershro, A. Proc. Natl. Acad. Sci. USA 1989, 86, 7751-7755.

27 Glickman, M. H., Rubin, D. M., Coux, O., Wefes, I., Pfeifer, G., Cjeka, Z., Baumeister, W., Fried, V. A. and Finley, D. Cell 1998, 94, 615-623.

28 Hadari, T., Warms, J. V. B., Rose, I. A. and Hershro, A. J. Biol. Chem. 1992, 267, 719-727. 
29 Eytan, E., Armon, T., Heller, H., Beck, S. and Hersh ko, A. J. Biol. Chem. 1993, 268, 4668-4674.

30 Verma, R., Aravind, L., OAnia, R., McDonald, W. H., Yates, W. R., Koonin, E. V. and Deshaies, R. J. Science 2002, 298, 611-615.

31 Finley, D., Ciechanover, A. and Varshavsky, A. Cell 1984, 37, 43-55.

32 Finley, D., Ozkanyak, E. and Varshavsky, A. Cell 1987, 48, 10351046.

33 Jentsch, S., McGrath, J. P. and VARshavsky, A. Nature 1987, 329, 131-134.

34 Goebl, M. G., Jochem, J., Jentsch, S., McGrath, J. P., Varshavsky, A. and Byers, B. Science 1988, 241, 13311335.

35 Evans, T., Rosenthal, E. T., Youngbloom, J., Distel, D. and Hunt, T. Cell 1983, 33, 289-396.

36 Glotzer, M., Murray, A. W. and KirSCHNER, M. W. Nature 1991, 349, 132-138.
37 Hershro, A., Ganoth, D., Pehrson, J., Palazzo, R. E. and Cohen, L. H. J. Biol. Chem. 1991, 266, 1627616379.

38 Hershio, A., Ganoth, D., Sudakin, V., Dahan, A., Cohen, L. H., Luca, F. C., Ruderman, J. and Eytan, E. J. Biol. Chem. 1994, 269, 49404946.

39 Sudakin, V., Ganoth, D., Dahan, A., Heller, H., Hershko, J., Luca, F. C., Ruderman, J. V. and Hershoo, A. Mol. Biol. Cell 1995, 6, 185-198.

40 King, R. W., Peters, J. M., Tugendreich, S., Rolfe, M., Hieter, P. and Kirschner, M. W. Cell 1995, 81, 279-288.

41 Irniger, S., Piatti, S., Michaelis, C. and Nasmyth, K. Cell 1995, 81, 269277.

42 Harper, J. W., Burton, J. C. and Solomon, M. J. Genes Dev. 2002, 16, 2179-2206.

43 Амоn, A. Curr. Op. Cell Biol. 1999, 9, 69-75. 